\title{
CDBA Based Universal Inverse Filter
}

\author{
Rajeshwari Pandey, ${ }^{1}$ Neeta Pandey, ${ }^{1}$ Tushar Negi, ${ }^{2}$ and Vivek Garg ${ }^{2}$ \\ ${ }^{1}$ Department of Electronics and Communication, Delhi Technological University, Bawana Road, Delhi 110042, India \\ ${ }^{2}$ Department of Electrical Engineering, Delhi Technological University, Bawana Road, Delhi 110042, India
}

Correspondence should be addressed to Rajeshwari Pandey; rajeshwaripandey@gmail.com

Received 30 November 2012; Accepted 3 February 2013

Academic Editors: H.-C. Chien, C. W. Chiou, and E. Tlelo-Cuautle

Copyright (C) 2013 Rajeshwari Pandey et al. This is an open access article distributed under the Creative Commons Attribution License, which permits unrestricted use, distribution, and reproduction in any medium, provided the original work is properly cited.

Current difference buffered amplifier (CDBA) based universal inverse filter configuration is proposed. The topology can be used to synthesize inverse low-pass (ILP), inverse high-pass (IHP), inverse band-pass (IBP), inverse band-reject (IBR), and inverse all-pass filter functions with appropriate admittance choices. Workability of the proposed universal inverse filter configuration is demonstrated through PSPICE simulations for which CDBA is realized using current feedback operational amplifier (CFOA). The simulation results are found in close agreement with the theoretical results.

\section{Introduction}

Inverse filters are commonly used in communication [1], speech processing, audio and acoustic systems $[2,3]$, and instrumentation [4] to reverse the distortion of the signal incurred due to signal processing and transmission. The transfer characteristics of the system that caused the distortion should be known a priori and the inverse filter to be used should have a reciprocal transfer characteristic so as to result in an undistorted desired signal. Literature review on inverse filter suggests that numerous well-established [5] methods for digital inverse filter design do exist but analog inverse filter design remained unexplored area as is evident from the limited availability of analog inverse filter circuits/design methods [6-14] until recently. However recent research trend suggests that the area is now gaining a renewed interest.

A brief account of the complete literature on analog inverse filter is presented here. Reference [6] presents a general method for obtaining the inverse transfer function for linear dynamic systems and the inverse transfer characteristic for nonlinear resistive circuits using nullors. The realization procedures for the current-mode FTFN-based inverse filters from the voltage-mode op-amp-based RC filters are presented in $[7,8]$. The procedure outlined in [7] is applicable to planar circuits only as it uses RC: CR dual transformation, whereas the method presented in [8] makes use of adjoint transformation and thus is applicable to nonplanar circuits
[12]. Single FTFN based inverse filters proposed in [9-12] present inverse filters using current feedback operational amplifier (CFOA). All the circuits presented in $[10,11]$ provide single inverse filter function; however [12] presents a topology which can realize inverse low-pass (ILP), inverse high-pass (IHP), and inverse band-pass (IBP) filter functions by appropriate admittance choice. In $[13,14]$ inverse all-pass (IAP) filters have been implemented using current difference transconductance amplifier (CDTA) and current conveyors, respectively. This study reveals that no universal inverse filter configuration has been proposed in the literature so far, to the best of the authors' knowledge. Therefore the aim of this paper is to present a current differencing buffered amplifier (CDBA) based universal inverse filter topology which realizes all five inverse filter functions, namely, ILP, IHP, IBP, IBR and IAP by appropriate admittance selection.

\section{Circuit Description}

Inherent wide bandwidth which is virtually independent of closed-loop gain, greater linearity, and large dynamic range are the key performance features of current mode technique [15]. The CDBA being a current processing analog building block inherits the advantages of current mode technique. In addition, it is free from parasitic capacitances [16] as its input terminals are internally grounded. Thus this active bolck is appropriate for high frequency operation. The circuit symbol 


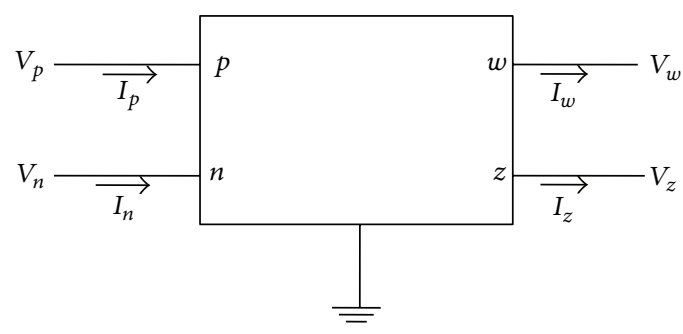

FIGURE 1: Block diagrammatic representation of CDBA.

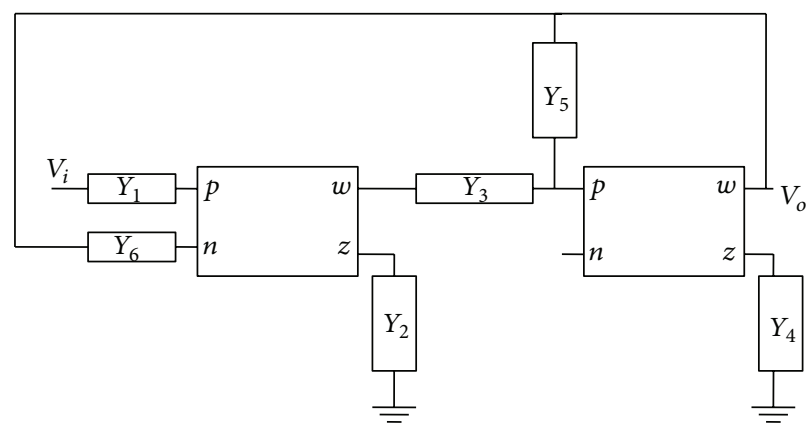

Figure 2: Proposed inverse filter configuration.

of CDBA is shown in Figure 1, and the port characteristics are given by

$$
\left[\begin{array}{c}
I_{z} \\
V_{w} \\
V_{p} \\
V_{n}
\end{array}\right]=\left[\begin{array}{cccc}
0 & 0 & 1 & -1 \\
1 & 0 & 0 & 0 \\
0 & 0 & 0 & 0 \\
0 & 0 & 0 & 0
\end{array}\right]\left[\begin{array}{c}
V_{z} \\
I_{w} \\
I_{p} \\
I_{n}
\end{array}\right]
$$

The proposed inverse filter configuration is shown in Figure 2. Routine analysis of the circuit of Figure 2 results in the following transfer function:

$$
\frac{V_{0}(s)}{V_{i}(s)}=\frac{N(s)}{D(s)}=\frac{Y_{1} Y_{3}}{Y_{2} Y_{4}+Y_{3} Y_{6}-Y_{2} Y_{5}}
$$

where

$$
\begin{aligned}
& N(s)=Y_{1} Y_{3}, \\
& D(s)=Y_{2} Y_{4}+Y_{3} Y_{6}-Y_{2} Y_{5} .
\end{aligned}
$$

With the admittance choices of $Y_{1}=s C_{1}+G_{1}$ and $Y_{3}=s C_{3}+$ $G_{3}$, the $N(s)$ can be expressed as

$$
N(s)=s^{2} C_{1} C_{3}+s\left(C_{1} G_{3}+C_{3} G_{1}\right)+G_{1} G_{3} .
$$

And the appropriate admittance choices for $Y_{2}, Y_{4}, Y_{5}$, and $Y_{6}$, as shown in Table 1 , would result in the required denominator functions $D(s)$ and hence the required inverse filter responses.
TABLE 1

\begin{tabular}{lcccc}
\hline Response type & $Y_{2}$ & $Y_{4}$ & $Y_{5}$ & $Y_{6}$ \\
\hline ILP & $G_{2}$ & $G_{4}$ & 0 & 0 \\
IHP & $s C_{2}$ & $s C_{4}$ & 0 & 0 \\
IBP & $s C_{2}$ & $G_{4}$ & 0 & 0 \\
IBR & $G_{2}$ & $G_{4}$ & $s C_{5}$ & $s C_{6}$ \\
IAP & $G_{2}$ & $G_{4}$ & $s C_{5}$ & $s C_{6}$ \\
\hline
\end{tabular}

Using the admittance choices given in Table 1, the ILP, IHP, and IBP response can, respectively, be expressed as

$$
\begin{aligned}
\frac{V_{0}}{V_{i}} & =\frac{1}{\left(G_{2} G_{4}\right) /\left(s^{2} C_{1} C_{3}+s\left(C_{1} G_{3}+C_{3} G_{1}\right)+G_{1} G_{3}\right)}, \\
\frac{V_{0}}{V_{i}} & =\frac{1}{\left(s^{2} C_{2} C_{4}\right) /\left(s^{2} C_{1} C_{3}+s\left(C_{1} G_{3}+C_{3} G_{1}\right)+G_{1} G_{3}\right)},
\end{aligned}
$$

$$
\frac{V_{0}}{V_{i}}=\frac{1}{\left(s C_{2} G_{4}\right) /\left(s^{2} C_{1} C_{3}+s\left(C_{1} G_{3}+C_{3} G_{1}\right)+G_{1} G_{3}\right)} \text {. }
$$

For admittance choices suggested for IBR and IAP in Table 1, the $D(s)$ can be written as

$$
D(s)=s^{2} C_{3} C_{6}+s\left(C_{6} G_{3}-C_{5} G_{2}\right)+G_{2} G_{5} .
$$

The resulting transfer function can be expressed as

$$
\frac{V_{0}(s)}{V_{i}(s)}=\frac{1}{\frac{s^{2} C_{3} C_{6}-s\left(C_{5} G_{2}-C_{6} G_{3}\right)+G_{2} G_{4}}{s^{2} C_{1} C_{3}+s\left(C_{1} G_{3}+C_{3} G_{1}\right)+G_{1} G_{3}}}
$$

which represents an IBR response if $C_{5}=C_{6}=C_{1}$ and $G_{1} G_{3}=G_{2} G_{5}$. The response will be IAP if $C_{5} G_{2}-C_{6} G_{3}=$ $C_{1} G_{3}+C_{3} G_{1}$ which can be easily obtained by choosing 


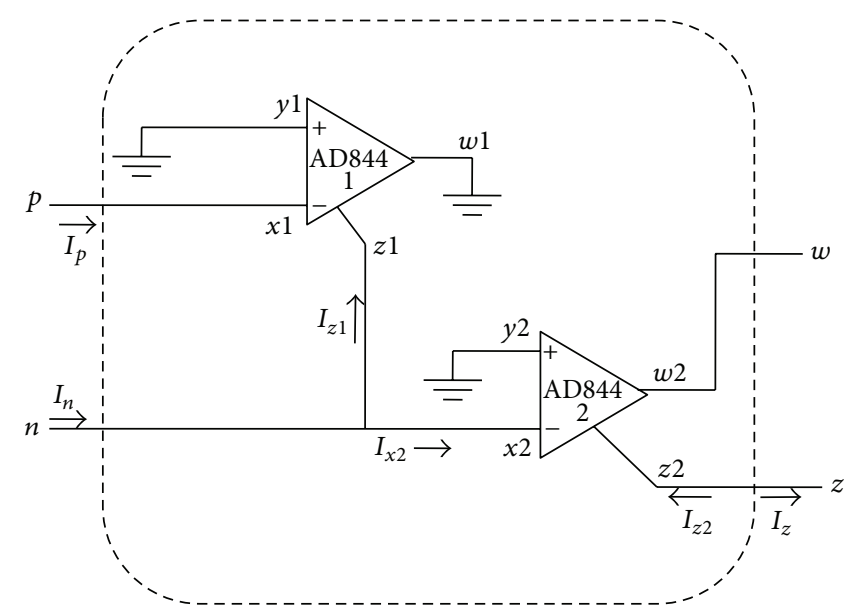

Figure 3: CDBA realization with AD844 [17].

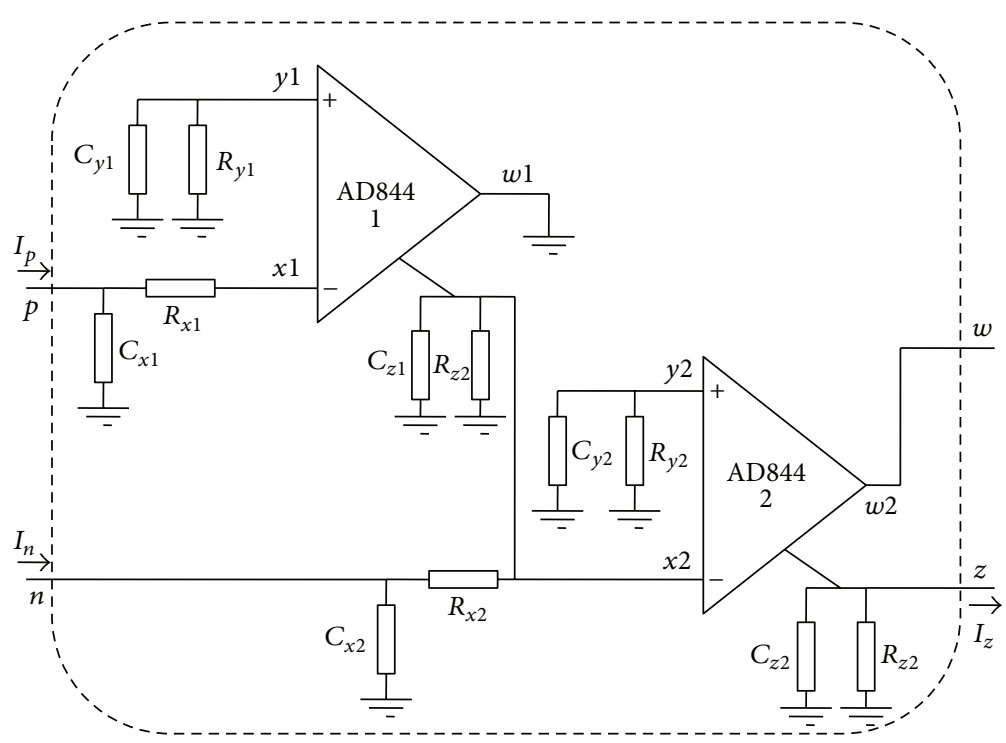

FIGURE 4: CDBA realization with nonideal model of AD844.

a suitable value of $C_{5}$. If $C_{3}=C_{6}=C_{1}=C$, then $C_{5}=3 C$ yields an IAP response provided $G_{1}=G_{2}=G_{3}$.

The resonant angular frequency $\left(\omega_{0}\right)$ and the quality factor $\left(Q_{0}\right)$ are given by (8) and (9), respectively, for all the responses

$$
\begin{aligned}
\omega_{0} & =\sqrt{\left(\frac{G_{1} G_{3}}{C_{1} C_{3}}\right)}, \\
Q_{0} & =\frac{\sqrt{C_{1} C_{3} G_{1} G_{3}}}{\left(C_{1} G_{3}+G_{1} C_{3}\right)},
\end{aligned}
$$

(whereas $\mathrm{H}_{\mathrm{ILP}}, \mathrm{H}_{\mathrm{IHP}}$, and $\mathrm{H}_{\mathrm{IBP}}$, the gain constants for ILP, IHP, and IBP responses, respectively, are given by

$$
\begin{gathered}
\mathrm{H}_{\mathrm{ILP}}=\frac{G_{2} G_{4}}{G_{1} G_{3}}, \quad \mathrm{H}_{\mathrm{IHP}}=\frac{C_{2} C_{4}}{C_{1} C_{3}}, \\
\mathrm{H}_{\mathrm{IBP}}=\frac{C_{2} G_{4}}{\left(C_{1} G_{3}+C_{3} G_{1}\right)} .
\end{gathered}
$$

\section{Sensitivity Analysis}

The passive sensitivities of $\omega_{0}$ and $Q_{0}$ for the proposed configuration can be expressed as

$$
\begin{aligned}
& S_{G_{1}}^{\omega_{0}}=S_{G_{3}}^{\omega_{0}}=\frac{1}{2}, \quad S_{G_{1}}^{\omega_{0}}=S_{C_{3}}^{\omega_{0}}=-\frac{1}{2}, \\
& S_{G_{1}}^{Q_{0}}=S_{C_{3}}^{Q_{0}}=\frac{1}{2}-\frac{C_{3} G_{1}}{\left(C_{1} G_{3}+C_{3} G_{1}\right)}, \\
& S_{G_{3}}^{Q_{0}}=S_{C_{1}}^{Q_{0}}=\frac{1}{2}-\frac{C_{1} G_{3}}{\left(C_{1} G_{3}+C_{3} G_{1}\right)} .
\end{aligned}
$$

It is clearly observed from (11) that the passive sensitivities are lower than $1 / 2$ in magnitude and hence the proposed universal inverse filter configuration may be termed as insensitive. 


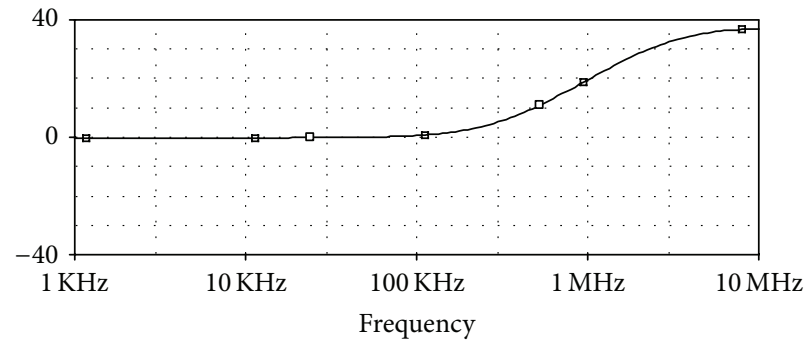

口 $\mathrm{DB}\left(V_{0}\right)$

(a)

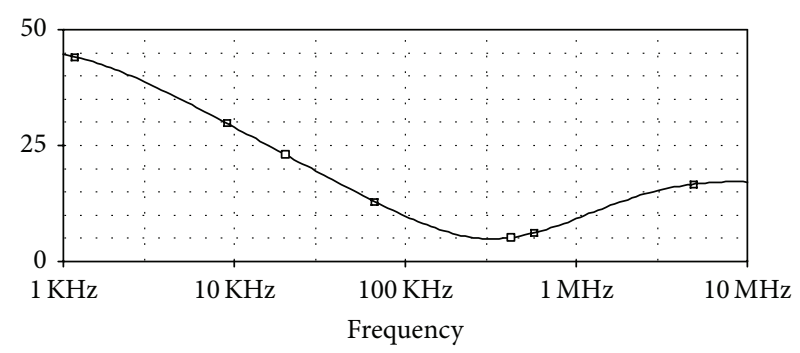

- $\mathrm{DB}\left(V_{0}\right)$

(c)

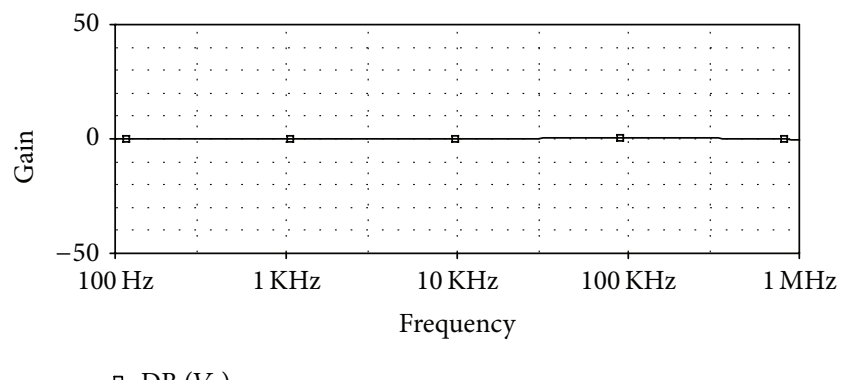

(e)

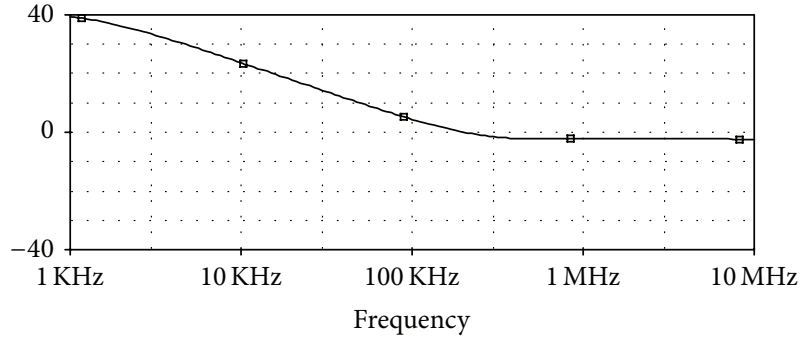

$\therefore \mathrm{DB}\left(V_{0}\right)$

(b)

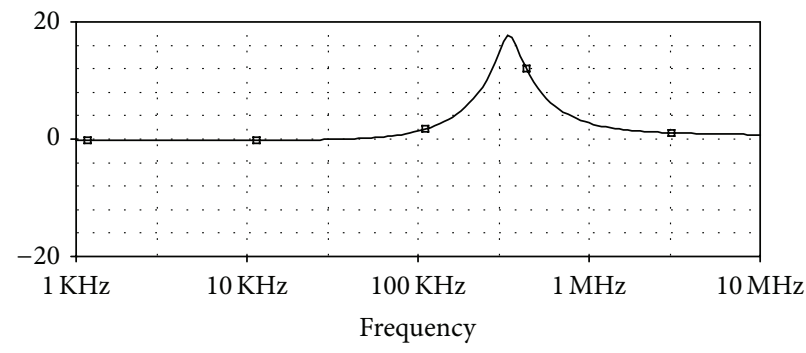

- $\mathrm{DB}\left(V_{0}\right)$

(d)

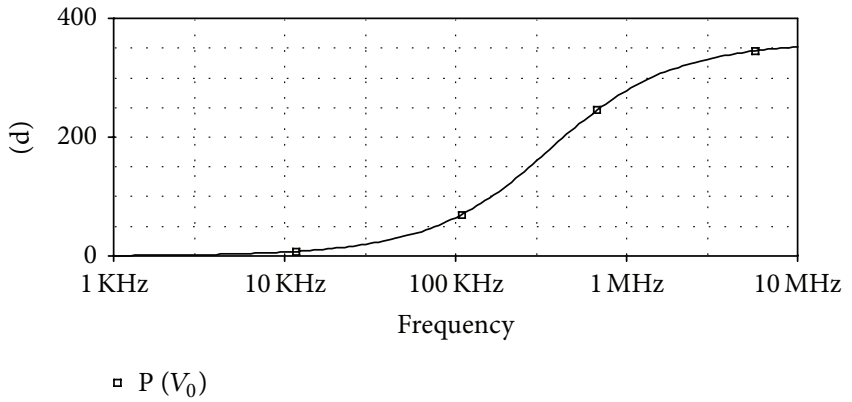

(f)

FiguRE 5: (a) Inverse low-pass response. (b) Inverse high-pass response. Inverse band-pass response. (d) Inverse band reject response. (e) Inverse all-pass magnitude response. (f) Inverse all-pass phase response.

\section{Realizing a CDBA and Associated Nonideality Analysis}

For the proposed configuration, the CDBA was realized using AD844 CFOA IC as shown in Figure 3 [17]. Ideally the input resistance at the $x$ terminal is zero and is infinite at the $z$ terminal. From Figure 3 various currents can be calculated as

$$
\begin{aligned}
& I_{z 1}=I_{p}, \\
& I_{x 2}=I_{n}-I_{z 1}, \\
& I_{z 2}=I_{x 2} .
\end{aligned}
$$

Therefore the current from $z$ terminal $I_{z}$ can be calculated as

$$
I_{z}=-I_{z 2}=\left(I_{p}-I_{n}\right) \text {. }
$$

And the output voltage $V_{w}$ is given as

$$
V_{w}=V_{z}
$$

In analysis so far, ideal characteristics of the CFOA have been considered. However, the effect of the parasitics of the CFOA needs to be taken into consideration for performing nonideality analysis [18-21]. For this, the model of AD844 [18] which includes a finite input resistance $R_{x}$ in series with $C_{x}$ at port- $x$, the $z$-port parasitic impedance $\left(R_{z} \| C_{z}\right)$, and the $y$-port parasitic impedance $\left(R_{y} \| C_{y}\right)$ is used. Using this nonideal model for CFOA, the CDBA structure of Figure 3 
modifies to Figure 4. The nonideal transfer function of ILP from Figure 4 can be expressed as

$$
\begin{aligned}
\frac{V_{0}}{V_{i}}=\left(s^{2} C_{1} C_{3}+s\left(C_{1} G_{3}+C_{3} G_{1}\right)+G_{1} G_{3}\right) / \\
G_{2}^{\prime} G_{4}^{\prime}\left(1+\frac{s C_{z 1}}{G_{2}^{\prime}}\right)\left(1+\frac{s C_{z 2}}{G_{4}^{\prime}}\right) \\
\times\left(1+\frac{s}{G_{x 1}}\left(\left(C_{x 1}+C_{1}\right)+G_{1}\right)\right) \\
\times\left(1+\frac{s}{G_{x 2}}\left(\left(C_{x 2}+C_{3}\right)+G_{3}\right)\right),
\end{aligned}
$$

where $G_{2}^{\prime}=1 /\left(R_{2} \| R_{z 1}\right)$ and $G_{4}^{\prime}=1 /\left(R_{4} \| R_{z 2}\right)$.

Considering $G_{x 1} \gg G_{2}$ and $G_{x 2} \gg G_{5},(15)$ modifies to

$$
\begin{aligned}
& \frac{V_{0}}{V_{i}}=\left(s^{2} C_{1} C_{3}+s\left(C_{1} G_{3}+C_{3} G_{1}\right)+G_{1} G_{3}\right) / \\
& G_{2}^{\prime} G_{4}^{\prime}\left(1+\frac{s C_{z 1}}{G_{2}^{\prime}}\right)\left(1+\frac{s C_{z 2}}{G_{4}^{\prime}}\right) \\
& \quad \times\left(1+\frac{s\left(G_{x 1}+C_{1}\right)}{G_{x 1}}\right)\left(1+\frac{s\left(G_{x 2}+C_{3}\right)}{G_{x 2}}\right) .
\end{aligned}
$$

It is clear from (16) that nonidealities of CFOA introduce parasitic poles in the transfer function. The deviation from the ideal behavior so caused can be kept small if all the external resistors are chosen to be much larger than $R_{x}$ but much smaller than $R_{y}$ and $R_{z}$. Similarly external capacitors should be chosen to be much larger than $C_{y}$ and $C_{z}$. Nonideal transfer functions for IHP and IBP can also be deduced in a similar manner.

\section{Simulation Results}

The proposed theoretical predictions are validated through simulations using PSPICE macromodel of CFOA AD844 IC as shown in Figure 3. Supply voltages used are $\pm 10 \mathrm{~V}$. The proposed inverse filter configuration is designed with equal value components. All the resistances of value $10 \mathrm{~K} \Omega$ and capacitors of value $50 \mathrm{pF}$ are chosen resulting in a theoretical $f_{0}$ of $318.5 \mathrm{KHz}$. Simulated frequency magnitude responses for ILP, IHP, IBP, IBR, and IAP are shown from Figure 5(a) to Figure 5(e), respectively, whereas Figure 5(f) shows the phase response for IAP. The simulated $f_{0}$ for all the responses is found to be $316.3 \mathrm{KHz}$ and is in close agreement to the theoretical value.

\section{Conclusion}

A current difference buffered amplifier (CDBA) based universal inverse filter configuration is proposed. Appropriate admittance selections allow using the proposed topology as one of the five inverse filter configurations, namely, ILP, IHP, IBP, IBR, and IAP. Workability of the proposed universal inverse filter configuration is demonstrated through PSPICE simulations for which CDBA is realized using current feedback operational amplifier (CFOA). The simulation results are found in close agreement with the theoretical results.

\section{References}

[1] J. K. Tugnait, "Identification and deconvolution of multichannel linear non-Gaussian processes using higher order statistics and inverse filter criteria," IEEE Transactions on Signal Processing, vol. 45, no. 3, pp. 658-672, 1997.

[2] A. Watanabe, "Formant estimation method using inverse-filter control," IEEE Transactions on Speech and Audio Processing, vol. 9, no. 4, pp. 317-326, 2001.

[3] O. Kirkeby and P. A. Nelson, "Digital filter design for inversion problems in sound reproduction," Journal of the Audio Engineering Society, vol. 47, no. 7-8, pp. 583-595, 1999.

[4] Z. Zhang, D. Wang, W. Wang, H. Du, and J. Zu, "A group of inverse filters based on stabilized solutions of fredholm integral equations of the first kind," in Proceedings of the IEEE International Instrumentation and Measurement Technology Conference, pp. 668-671, May 2008.

[5] G. John Proakis and G. Dimitris Manolakis, Digital Signal Processing, Prentice Hall, New York, NY, USA, 4th edition, 2007.

[6] A. Leuciuc, "Using nullors for realisation of inverse transfer functions and characteristics," Electronics Letters, vol. 33, no. 11, pp. 949-951, 1997.

[7] B. Chipipop and W. Surakampontorn, "Realization of currentmode FTFN-based inverse filter," Electronics Letters, vol. 35, no. 9, pp. 690-692, 1999.

[8] H. Y. Wang and C. T. Lee, "Using nullors for realisation of current-mode FTFN-based inverse filters," Electronics Letters, vol. 35, no. 22, pp. 1889-1890, 1999.

[9] M. T. Abuelma'atti, "Identification of cascadable current-mode filters and inverse-filters using single FTFN," Frequenz, vol. 54, no. 11-12, pp. 284-289, 2000.

[10] S. S. Gupta, D. R. Bhaskar, R. Senani, and A. K. Singh, "Inverse active filters employing CFOAs," Electrical Engineering, vol. 91, no. 1, pp. 23-26, 2009.

[11] S. S. Gupta, D. R. Bhaskar, and R. Senani, "New analogue inverse filters realised with current feedback op-amps," International Journal of Electronics, vol. 98, no. 8, pp. 1103-1113, 2011.

[12] H.-Y. Wang, S.-H. Chang, T.-Y. Yang, and P.-Y. Tsai, "A novel multifunction CFOA-based inverse filter," Circuits and Systems, vol. 2, pp. 14-17, 2011.

[13] N. A. Shah, M. Quadri, and S. Z. Iqbal, "High output impedance current-mode allpass inverse filter using CDTA," Indian Journal of Pure and Applied Physics, vol. 46, no. 12, pp. 893-896, 2008.

[14] N. A. Shah and M. F. Rather, "Realization of voltage-mode CCII-based allpass filter and its inverse version," Indian Journal of Pure and Applied Physics, vol. 44, no. 3, pp. 269-271, 2006.

[15] C. Toumazu, F. J. Lidgey, and D. G. Haigh, Analogue IC Design: The Current Mode Approach, IEEE Circuits and Systems Series, Peringrinus, 1990.

[16] C. Acar and S. Ozoguz, "A new versatile building block: current differencing buffered amplifier suitable for analog signalprocessing filters," Microelectronics Journal, vol. 30, no. 2, pp. 157-160, 1999.

[17] S. Özcan, A. Toker, C. Acar, H. Kuntman, and O. Çiçekoglu, "Single resistance-controlled sinusoidal oscillators employing current differencing buffered amplifier," Microelectronics Journal, vol. 31, no. 3, pp. 169-174, 2000. 
[18] AD844 Current Feedback Op-Amp Data Sheet, Analog Devices Inc., Norwood, NJ, USA, 1990.

[19] C. Sánchez-López, F. V. Fernández, E. Tlelo-Cuautle, and S. X. D. Tan, "Pathological element-based active device models and their application to symbolic analysis," IEEE Transactions on Circuits and Systems I, vol. 58, no. 6, pp. 1382-1395, 2011.

[20] E. Tlelo-Cuautle, C. Sánchez-López, and D. Moro-Frías, "Symbolic analysis of (MO)(I)CCI(II)(III)-based analog circuits," International Journal of Circuit Theory and Applications, vol. 38, no. 6, pp. 649-659, 2010.

[21] R. Trejo-Guerra, E. Tlelo-Cuautle, C. Sánchez-López, J. M. Muñoz-Pacheco, and C. Cruz-Hernández, "Realization of multiscroll chaotic attractors by using current-feedback operational amplifiers," Revista Mexicana de Fisica, vol. 56, no. 4, pp. 268$274,2010$. 

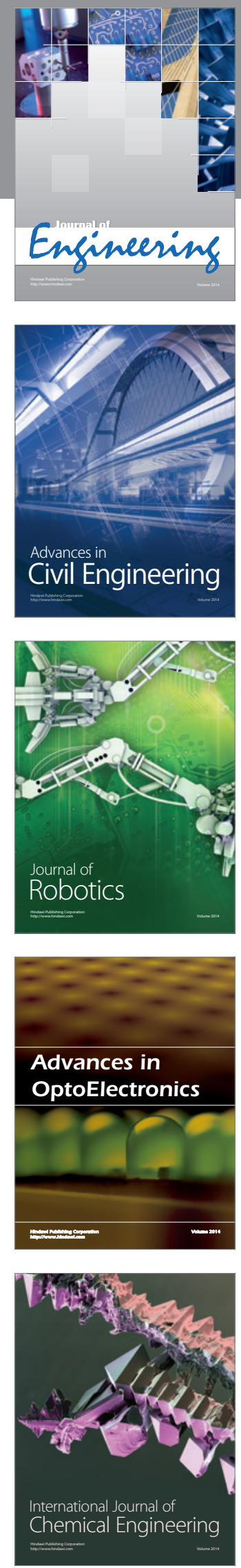

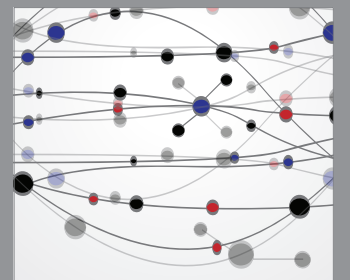

The Scientific World Journal
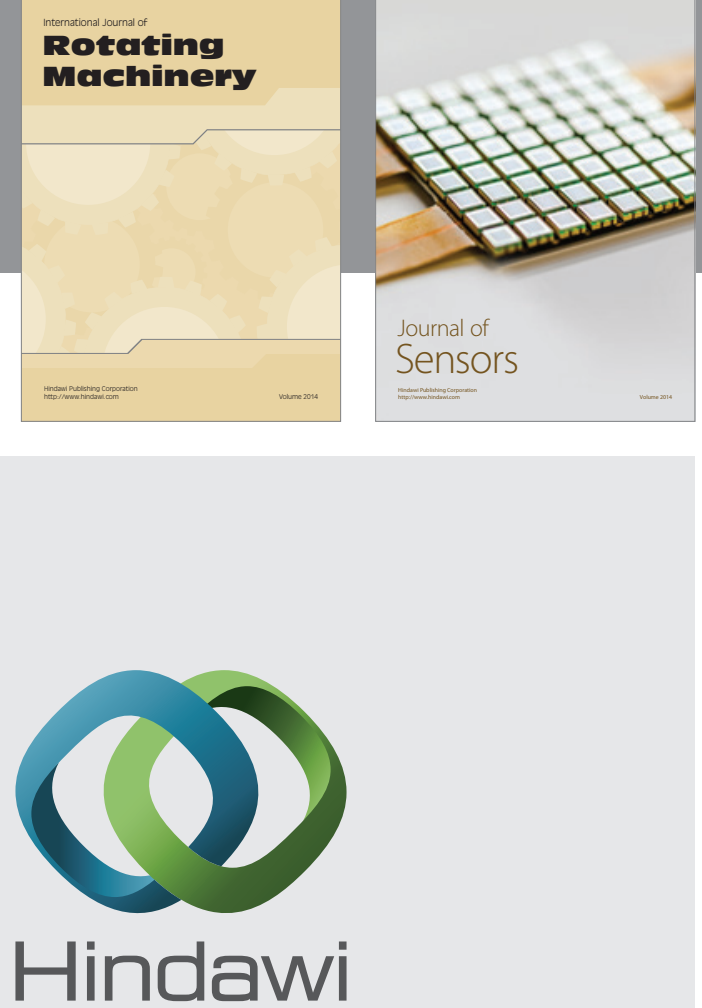

Submit your manuscripts at http://www.hindawi.com
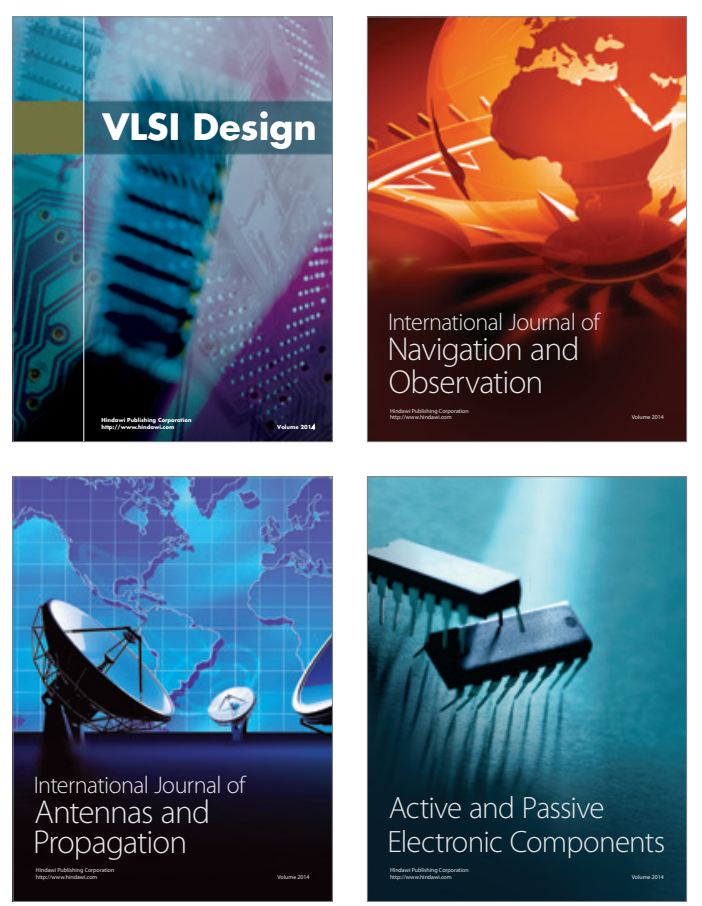
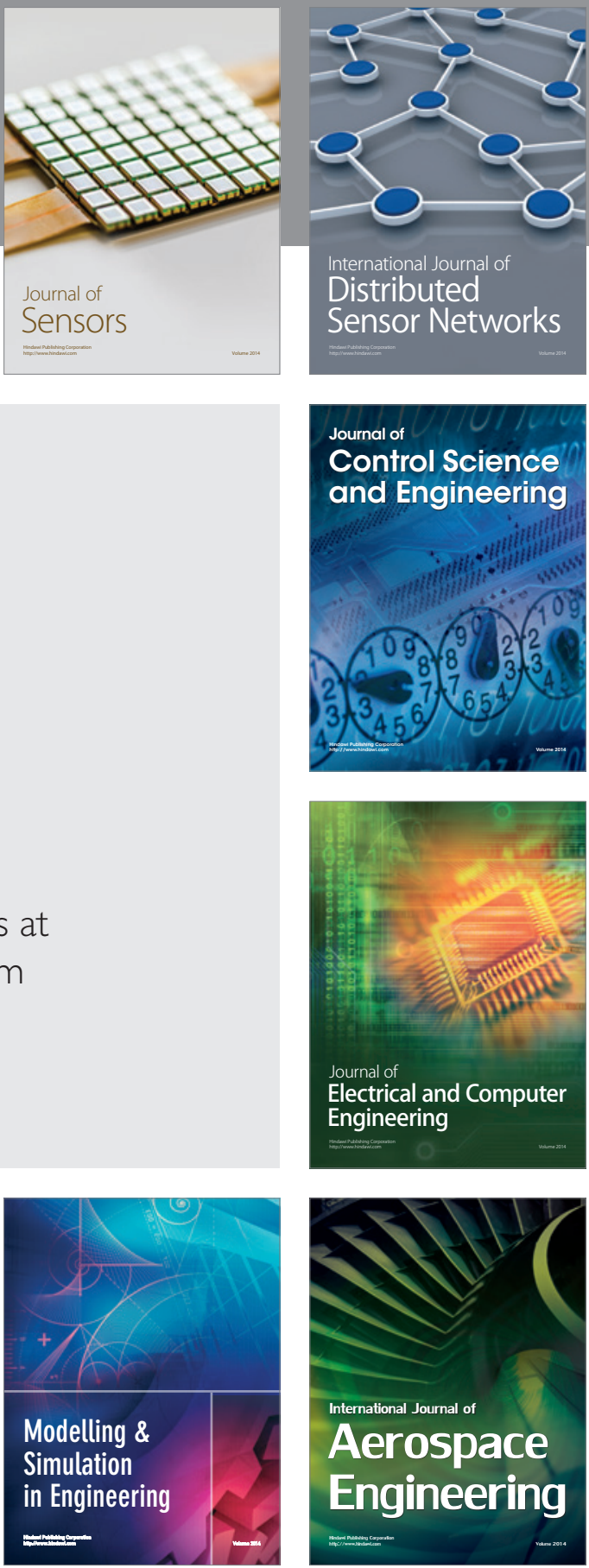

Journal of

Control Science

and Engineering
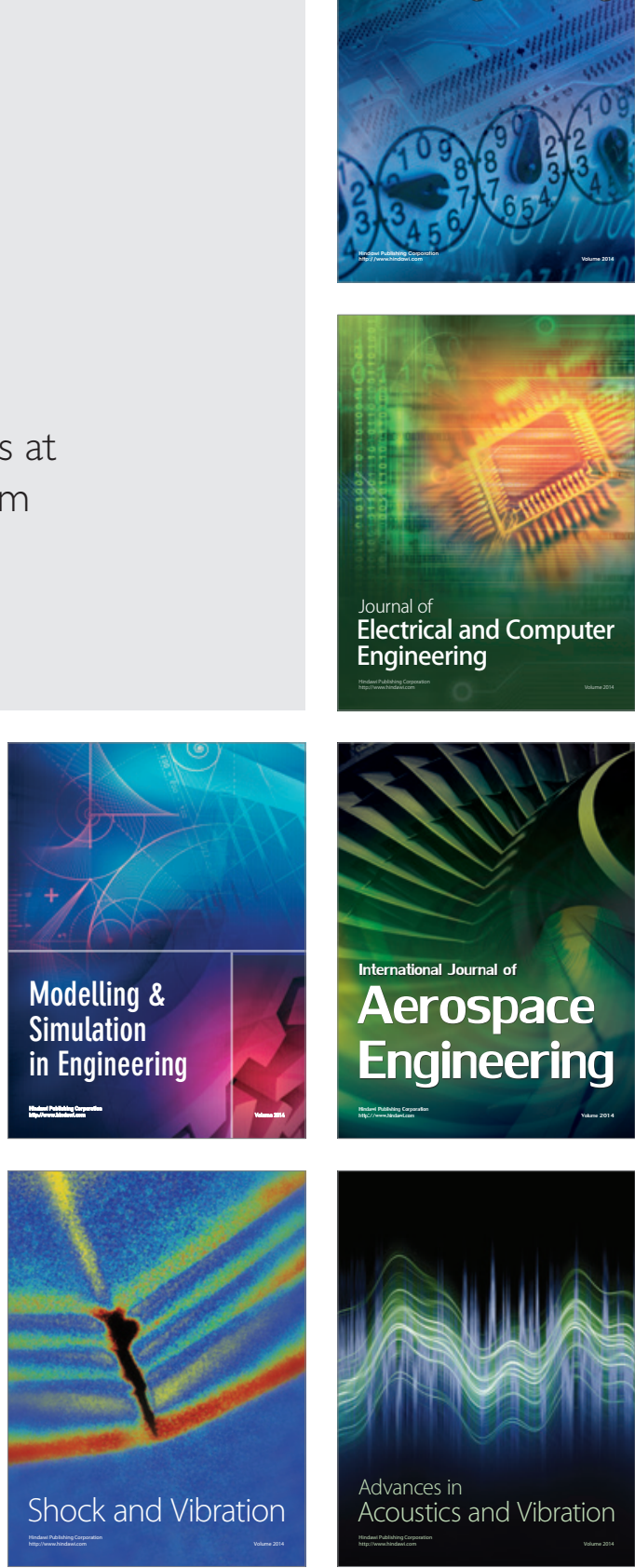